\title{
A NEW MATHEMATICAL MODEL FOR CLUSTER CALCULATION IN TETRAHEDRALLY BONDED AMORPHOUS SEMICONDUCTORS
}

\author{
M.A. GRADO CAFFARO \\ Consultant, Madrid (SPAIN) \\ M. GRADO CAFFARO \\ CSIC, Madrid (SPAIN)
}

(Received April 3, 1993; in final form April 27, 1993)

\begin{abstract}
Tetrahedrally bonded amorphous semiconductors exhibit a structure suitable for applying the so-called extended Hückel theory (EHT) to determine the electronic density of states by means of the cluster calculation. In the following, a new mathematical model based on EHT is established. The results obtained from this model have sufficient accuracy for applications and provide a useful methodology that combines adequately with the spectrum computations. Density of valence states has been obtained experimentally with monochromatized X-rays and the theoretical results obtained in this paper are in good agreement with these experiments.
\end{abstract}

\section{INTRODUCTION}

It is well-known that photoemission experiments have provided much information on the electronic spectra of tetrahedrally bonded amorphous semiconductors [1], [2], [3]. In this context, the density of valence states plays an important role. Moreover, for III-V compounds, the results obtained from photoemission experiments involving the energy margin within a few $\mathrm{eV}$ of the band-gap are related to their optical properties. In particular, the $\xi_{2}$-spectra of amorphous InSb cannot be obtained by broadening that of the crystal [4].

On the other hand, in order to determine the electronic density of states in semiconductors with spatial disorder, the "cluster calculation" [5] is feasible. In this paper, we will develop a method based on EHT which, in principle, consists of the decomposition of a cluster in sub-clusters and further calculation of the density value of all sub-cluster contributions.

\section{PRELIMINARIES}

By using EHT, the local electronic density of states for the semiconductors in question can be expressed as follows ([5]):

$\mathrm{g}(\overrightarrow{\mathrm{r}}, \mathrm{E})=-\frac{1}{\pi} \operatorname{ImG}(\overrightarrow{\mathrm{r}}, \mathrm{E})=\sum\left|\psi_{\mathrm{n}}(\overrightarrow{\mathrm{r}})\right|^{2} \delta\left(\mathrm{E}-\mathrm{E}_{\mathrm{n}}\right)$ 
where $\mathrm{G}$ is the Green's function, E denotes energy, $\overrightarrow{\mathrm{r}}$ denotes position vector, $\delta$ is the Dirac function, and $\psi_{\mathrm{n}}$ are the eigen-wavefunctions associated with the energy eigenvalues $E_{n}$.

In order to calculate the density of states in materials with spatial disorder, the cluster calculation is adequate for this purpose ([5]). In the following, we will assume a cluster that is isolated in vacuum. The density of states of the cluster is given by

$\tilde{g}(E)=\iiint_{D} g(x, y, z, E) d x d y d z$

with respect to cartesian coordinates. $\mathrm{D}$ denotes the domain corresponding to the cluster. Now, we establish the following approximation by means of a Taylor-Mac Laurin series:

$\mathrm{g}(\overrightarrow{\mathrm{r}}, \mathrm{E}) \approx \mathrm{g}(\overrightarrow{\mathrm{o}}, \mathrm{E})+\overrightarrow{\mathrm{r}} \cdot\left[\nabla_{\vec{r}} \mathrm{~g}\right]_{0}$

where $\nabla_{\vec{r}} \mathrm{~g}$ refers to the spatial coordinates, that is,

$\nabla_{\vec{r}} \equiv\left(\frac{\partial}{\partial x}, \frac{\partial}{\partial y}, \frac{\partial}{\partial z}\right)$

By substituting (3) into (2) we obtain

$\tilde{\mathrm{g}}(\mathrm{E}) \approx \Omega \mathrm{g}(\overrightarrow{\mathrm{o}}, \mathrm{E})+\iiint_{\mathrm{D}} \overrightarrow{\mathrm{r}} \cdot\left[\nabla_{\vec{r}} \mathrm{~g}\right]_{0} \mathrm{dxdydz}$

where $\Omega$ is the volume of the cluster. Introducing the wavefunctions $\psi_{\mathrm{n}}$, from (1) we find

$\mathrm{g}(\overrightarrow{\mathrm{o}}, \mathrm{E})=\sum_{\mathrm{n}}\left|\psi_{\mathrm{n}}(\overrightarrow{\mathrm{o}})\right|^{2} \delta\left(\mathrm{E}-\mathrm{E}_{\mathrm{n}}\right)$

$\nabla_{\vec{r}} \mathrm{~g}=2 \sum_{\mathrm{n}}\left\{\left[\operatorname{Re} \psi_{\mathrm{n}}\right] \nabla\left[\operatorname{Re} \psi_{\mathrm{n}}\right]+\left[\operatorname{Im} \psi_{\mathrm{n}}\right] \nabla\left[\operatorname{Im} \psi_{\mathrm{n}}\right]\right\} \delta\left(\mathrm{E}-\mathrm{E}_{\mathrm{n}}\right)$

REMARK: In the previous exposition, the point $0=(0,0,0)$ is the origin of the cluster.

By substituting (5) and (6) into (4), we obtain

$$
\begin{aligned}
\tilde{\mathbf{g}}(\mathrm{E})=\sum_{\mathrm{n}}\left\{\Omega\left|\psi_{\mathrm{n}}(\overrightarrow{\mathrm{o}})\right|^{2}+2 \iiint_{\mathrm{D}} \overrightarrow{\mathrm{r}} \cdot\left[\operatorname{Re}\left(\psi_{\mathrm{n}}\right)_{0}\left(\nabla \operatorname{Re}\left(\psi_{\mathrm{n}}\right)\right)_{0}\right.\right. \\
\left.\left.+\operatorname{Im}\left(\psi_{\mathrm{n}}\right)_{0}\left(\nabla \operatorname{Im}\left(\psi_{\mathrm{n}}\right)\right)_{0}\right] \mathrm{dxdydz}\right\} \delta\left(\mathrm{E}-\mathrm{E}_{\mathrm{n}}\right)
\end{aligned}
$$




\section{CALCULATIONS RELATED TO $\xi_{2}$-SPECTRUM}

$\xi_{2}$-spectra have been extensively studied [6], [7], [8], [9]. Now, we assume a symmetric density of states spectrum with overlapping valence and conduction band tails and with mobility edges in accordance with ref. [6]. Moreover, we consider exponential band tails. Under these conditions, we formulate the following relationship [6], [7]:

$\xi_{2}(\omega) \approx C \omega^{-2} \int_{0}^{\hbar \omega} \tilde{\mathrm{g}}(\mathrm{E}) \tilde{\mathrm{g}}(\hbar \omega-\mathrm{E}) \mathrm{dE}$

where $\mathrm{C}$ is a constant and $\hbar \omega>2 \mathrm{E}_{\mathrm{C}}\left(\mathrm{E}_{\mathrm{C}}\right.$ is the conduction band mobility edge). If $\mathrm{E}_{\mathrm{C}}<\hbar \omega<2 \mathrm{E}_{\mathrm{C}}$ ( $\hbar \omega$ is photon energy), the relationship for optical transitions is as follows [6]

$\xi_{2}(\omega) \approx 2 \mathrm{C} \omega^{-2} \int_{\mathrm{E}_{\mathrm{C}}}^{\hbar \omega} \tilde{\mathrm{g}}(\mathrm{E}) \tilde{\mathrm{g}}(\hbar \omega-\mathrm{E}) \mathrm{dE}$

Expressions (8) and (9) are established according to the following claims:

1.- The states below $E_{V}$ and above $E_{C}$ are extended while the states between $E_{\mathrm{V}}$ and $\mathrm{E}_{\mathrm{C}}$ are localized.

2.- Extended to local transitions, local to extended transitions, and extended to extended transitions all have the same matrix elements.

3.-Local to local transitions are rigorously forbidden.

On the other hand, Equation (8) can be expressed as a convolution, namely:

$\xi_{2}(\omega) \approx \mathrm{C} \omega^{-2}[\tilde{\mathrm{g}}(\hbar \omega)] *[\tilde{\mathrm{g}}(\hbar \omega)]$

Applying Borel convolution theorem for the Laplace transform, we have:

$\hbar^{-2} £\left[(\hbar \omega)^{2} \xi_{2}(\hbar \omega)\right]=C\{£[\tilde{g}(\hbar \omega)]\}^{2}$

where $£$ denotes Laplace operator. Next, we will calculate the left-hand side of (11). The result is:

$\hbar^{-2} £\left[(\hbar \omega)^{2} \xi_{2}(\hbar \omega)\right]=\frac{\mathrm{d}^{2}}{\mathrm{ds}^{2}}\left\{£\left[\xi_{2}(\hbar \omega)\right]\right\}$

From (11) and (12) it is derived:

$\tilde{\mathrm{g}}(\hbar \omega)=\mathrm{C}^{-1 / 2} \mathfrak{f}^{-1}\left\{\frac{\mathrm{d}^{2}}{\mathrm{ds}^{2}}\left[\mathfrak{f}\left(\xi_{2}(\hbar \omega)\right)\right]\right\}^{1 / 2} \quad\left(\hbar \omega>2 \mathrm{E}_{\mathrm{C}}\right)$

By means of Equation (13), it is feasible to obtain the density of states when the $\xi_{2}$-spectrum is known from experimental works. 


\section{SUBCLUSTER APPROACH}

Considering a given cluster, it is obvious that the approximation $g(\overrightarrow{\mathrm{r}}, \mathrm{E}) \approx \mathrm{g}(\overrightarrow{\mathrm{o}}, \mathrm{E})$ is crude. However, this approximation is admissible if we consider a neighborhood around the point in question. If we make a decomposition of a cluster to obtain neighborhoods around all the points of the cluster, then the neighborhoods are called subclusters or very small zones belonging to the cluster. Under these conditions, we choose a number of points belonging to the cluster whose respective position vectors are $\overrightarrow{\mathbf{r}}_{1}, \overrightarrow{\mathbf{r}}_{2}, \ldots, \overrightarrow{\mathbf{r}}_{\mathrm{N}}$. Then, for a generic sub-cluster $\left(\mathrm{k}^{\text {th }}\right.$ sub-cluster $)$, we consider the zero-th order Taylor expansion:

$\left.\mathrm{g}(\overrightarrow{\mathrm{r}}, \mathrm{E})\right|_{\mathrm{k}} \approx \mathrm{g}\left(\overrightarrow{\mathrm{r}}_{\mathrm{k}}, \mathrm{E}\right)$

Therefore, the density of states of the $\mathrm{k}^{\text {th }}$ sub-cluster in question is given by (see eq. (2))

$\overrightarrow{\mathrm{g}}_{\mathrm{k}}(\mathrm{E}) \approx \Omega_{\mathrm{k}} \mathrm{g}\left(\overrightarrow{\mathrm{r}}_{\mathrm{k}}, \mathrm{E}\right)$

where $\Omega_{\mathrm{k}}$ is the volume of the sub-cluster. Finally, the total density of states in the cluster is given by the following expression:

$\tilde{g}(E) \approx\left(\sum_{k=1}^{N} \Omega_{k} g\left(\vec{r}_{k}, E\right)\right) / N$

with: $\Omega=\sum_{\mathrm{k}=1}^{\mathrm{N}} \Omega_{\mathrm{k}}$.

By (1) we have:

$\tilde{g}(E) \approx\left\{\sum_{k} \Omega_{k}\left[\sum_{n}\left|\psi_{n}\left(\vec{r}_{k}\right)\right|^{2} \delta\left(E-E_{n}\right)\right]\right\} / N$

that is,

$\tilde{\mathrm{g}}(\mathrm{E}) \approx \sum_{\mathrm{n} . \mathrm{k}}^{\mathrm{N}} \mathrm{a}_{\mathrm{nk}} \zeta_{\mathrm{n}} \Omega_{\mathrm{k}}$

where $a_{n k} \equiv\left|\psi_{n}\left(\vec{r}_{k}\right)\right|^{2} N^{-1}$, and $\delta\left(E-E_{n}\right) \equiv \zeta_{n}$. We assume that each subcluster contains one atom only, whose position vector is $\vec{r}_{k}(k=1,2, \ldots, N)$.

Therefore, the total density of states for a given cluster can be determined by eq. (18). This formula can be connected with eq. (13) so that $\tilde{\mathrm{g}}(\hbar \omega)$ is identified as $\tilde{g}(E)$ by replacing $E$ with $\hbar \omega$ in (18), as follows:

$\frac{d^{2}}{d s^{2}}\left[£\left(\xi_{2}(\hbar \omega)\right)\right]=C\left\{\sum_{n, k}^{N} a_{n k} \Omega_{k} f\left[\delta\left(\hbar \omega-E_{n}\right)\right]\right\}^{2}$ 
By taking into account

$f\left[\delta\left(\hbar \omega-E_{n}\right)\right]=\int_{0}^{\infty} \delta\left(\hbar \omega-E_{n}\right) \exp (-\hbar \omega s) d(\hbar \omega)=\exp \left(-s E_{n}\right)$

eq. (19) becomes

$\xi_{2}(\hbar \omega)=C £^{-1} \int\left(\int\left(\sum_{n, k}^{N} a_{n k} \Omega_{k} \exp \left(-s E_{n}\right)\right)^{2} d s\right) d s$

with $\hbar \omega>2 \mathrm{E}_{\mathrm{C}}$.

\section{CONCLUSIONS}

The previous methodology represents a substantial improvement of the theory on atom-clusters in tetrahedrally bonded amorphous semiconductors. The cluster calculation refers to numerical methods that are suitable for computer programs and plays a relevant role.

However, cluster calculation from the point of view of mathematical physics should be improved in elegant ways. It is convenient to elaborate new formulations since several questions remain open. The purpose of the work developed here consists of the improvement of cluster theory by means of the sub-cluster approach, which implies calculations that lead to eq. (18).

In addition, with respect to $\xi_{2}$-spectrum, eq. (20) represents an interesting result. The relation between infrared absorption spectrum and cluster approach has been analyzed implicitly by M.A. Grado and M. Grado [7].

\section{REFERENCES}

1. N.J. Shevchik. AIP Conf. Proc. no. 20, (1974), 72-84.

2. D. Brust. Phys. Rev. 186, (1969), 768.

3. D.E. Polk. J. Non-Cryst. Solids 5, (1971), 365.

4. N.J. Shevchik, W. Paul. J. Non-Cryst. Solids 13, (1973), 1.

5. B.Y. Tong. AIP Conf. Proc. no. 20, (1974), 148.

6. H.K. Rockstad. AIP Conf. Proc. no. 20, (1974), 18-26.

7. M.A. Grado, M. Grado, Phys. Lett A, 169, no. 5, (1992), 399-401.

8. N.K. Hindley. J. Non-Cryst. Solids, 5, (1970), 17.

9. S.S. Mitra, Y.F. Tsay, D.K. Paul, B. Bendow. Phys. Rev. B, 15, (1977), 4007. 

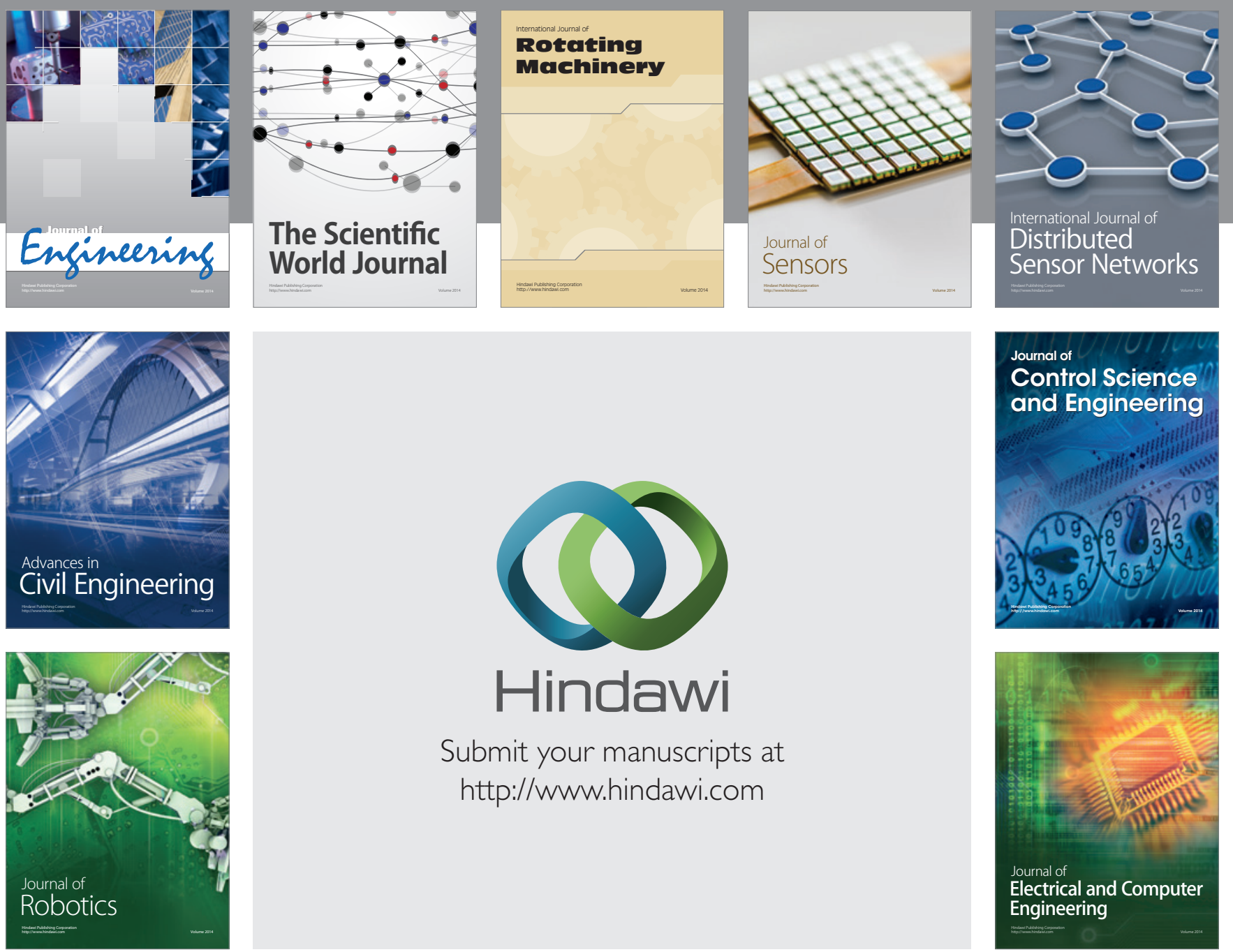

Submit your manuscripts at

http://www.hindawi.com
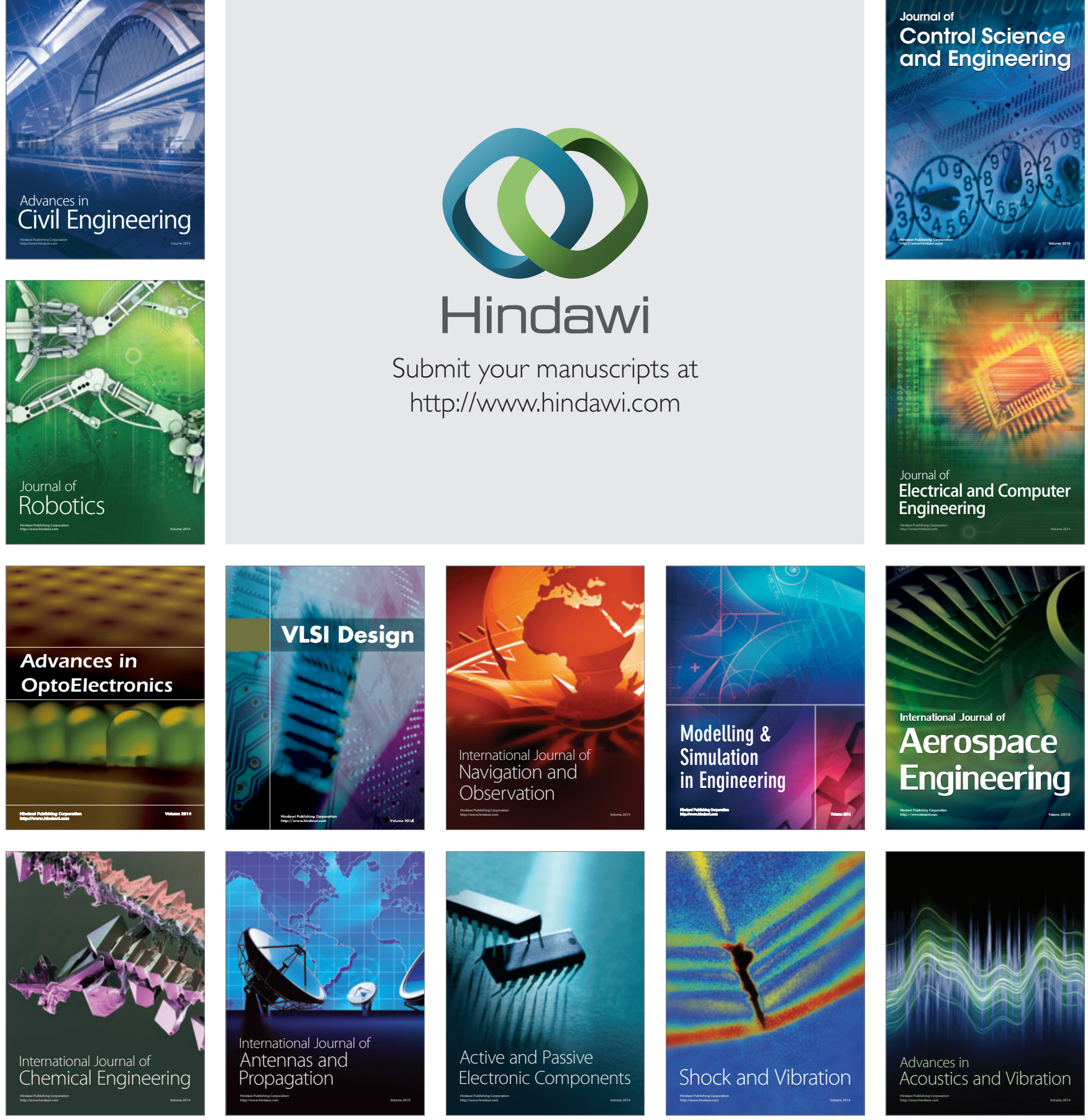